SYNLETT

Spotlight 408

This feature focuses on a reagent chosen by a postgraduate, highlighting the uses and preparation of the reagent in current research

\section{Cyclopropenium Ion}

Compiled by Arvind Kumar Yadav

Arvind Kumar Yadav was born in Ghazipur, U.P., India. He received his M.Sc. degree (2010) in chemistry from the University of Allahabad, India. In the same year, he joined the research group of Prof. L. D. S. Yadav as a PhD student. He has qualified twice for a Junior Research Fellowship (JRF) in the National Eligibility Test (NET) of CSIR-UGC in 2010 and 2011. His research is focused on organic synthesis via organocatalysis.

Green Synthesis Lab, Department of Chemistry, University of Allahabad, Allahabad 211002, U.P. India

E-mail: arvindkumaryadav@hotmail.co.in

Dedicated to my honorable mentor Prof. L. D. S. Yadav

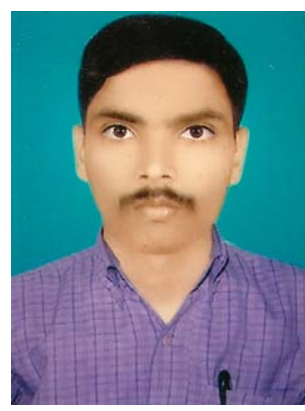

\section{Introduction}

The cyclopropenium ion, an aromatic cation, is the smallest member of the Hückel aromatic systems. It was first prepared by Breslow in 1957 as triphenylcyclopropenium perchlorate. ${ }^{1}$ Despite the apparent inherent strain in the cyclopropene ring, cyclopropenium ions possess considerable thermodynamic stability and exhibit the dual properties of stability and ionic charge. Thus, they easily combine with an anion or a Lewis basic heteroatom leading to the reversible generation of the corresponding neutral carbocyclic species (Scheme 1). Owing to their unique reactivity, cyclopropenium ions, especially 2,3 -diarylcyclopropenium ions, have been strategically exploited for the activation of the hydroxyl group of alcohols, carboxylic acids, oximes, and diols in various synthetically useful dehydrative processes to afford alkyl chlorides, ${ }^{2,3}$ acid chlorides, ${ }^{4}$ amides/lactams, ${ }^{5,6}$ and cyclic ethers, respectively. ${ }^{7}$

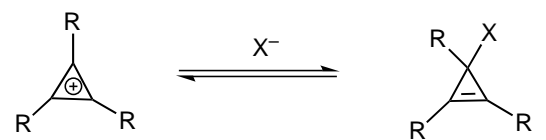

Scheme 1 Cyclopropenium ion

\section{Preparation}

The diarylcyclopropenium ions, frequently used in synthetically important organic transformations, are readily prepared by the action of thionyl chloride or oxalyl chloride on 2,3-diarylcyclopropenones (Scheme 2). ${ }^{2,8}$

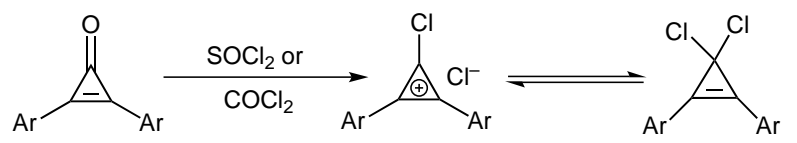

Scheme 2 Preparation of cyclopropenium ions

\title{
Abstracts
}

(A) Conversion of Alcohols into Alkyl Chlorides:

Kelly and Lambert have reported a convenient and efficient method for converting alcohols into alkyl chlorides. ${ }^{2}$ The protocol represents a novel paradigm for alcohol activation for the promotion of substitution reactions employing a cyclopropenium ion. Furthermore, the same research group has very recently reported a cyclopropenonecatalyzed conversion of alcohols into alkyl chlorides involving the cyclopropenium ion activation. ${ }^{3}$

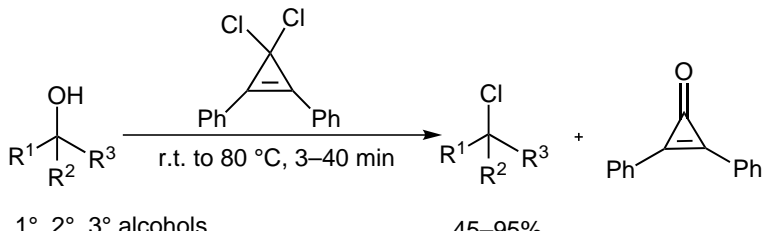

$1^{\circ}, 2^{\circ}, 3^{\circ}$ alcohols $45-95 \%$
(B) Conversion of Carboxylic Acids into Acid Chlorides: Expanding beyond the alcohol activation with a cyclopropenium ion, Lambert and co-workers have shown that a similar methodology can be applied to the activation of acid chlorides. ${ }^{4}$

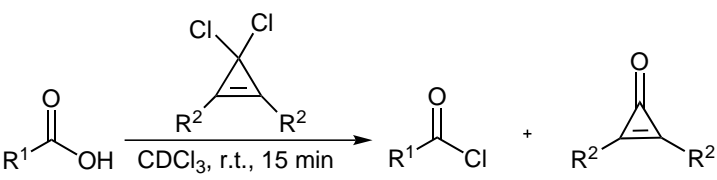

SYNLETT 2012, 23, 2428-2429

Advanced online publication: 13.09.2012

DOI: 10.1055/s-0032-1317230; Art ID: ST-2012-V0415-V

(C) Georg Thieme Verlag Stuttgart · New York 
(C) Beckmann Rearrangement:

Yadav and co-workers have recently reported a cyclopropenium ion catalyzed (3 mol\% loading) Beckmann rearrangement of ketoximes to afford the corresponding amides/lactams within two hours. ${ }^{5}$ This is the first example of cyclopropenium ion catalysis.

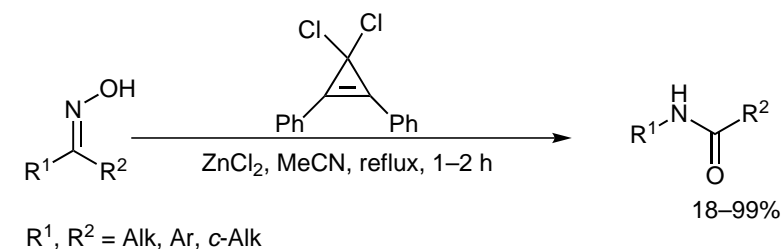

(D) Cyclodehydration of Diols:

Building upon their earlier work on chlorodehydration, Kelley and Lambert have developed a cyclopropenium ion activated cyclodehydration of 1,4- and 1,5-diols to the corresponding cyclic ethers. ${ }^{7}$

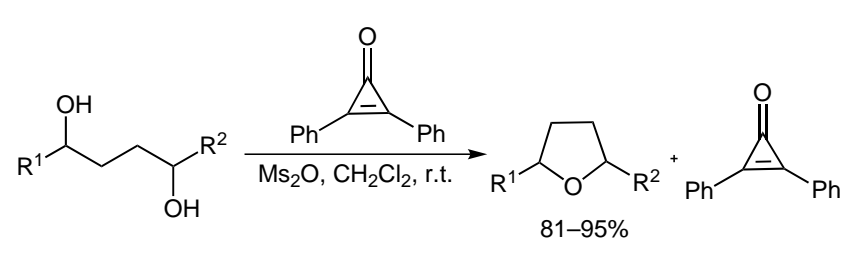

(E) Dehydrative Glycosylation:

Bennett and co-workers have reported cyclopropenium ion promoted dehydrative glycosylation reactions of 2-deoxy and 2,6-dideoxy sugars. The reactions are $\alpha$-selective and proceed under mild conditions at room temperature without the need of special dehydrating agents. ${ }^{9}$

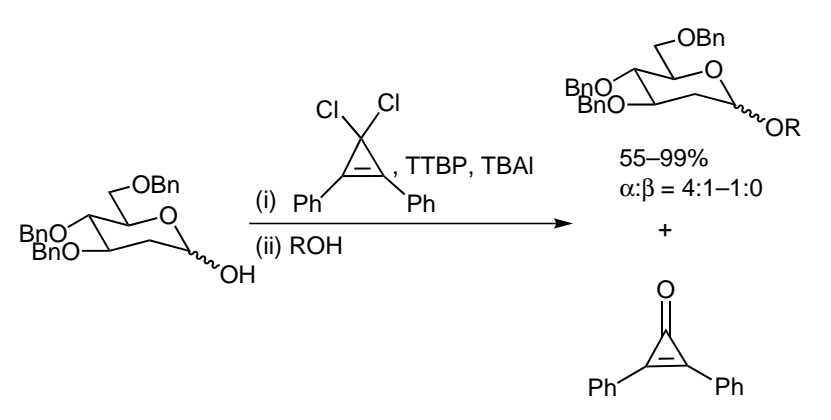

\section{References}

(1) Breslow, R. J. Am. Chem. Soc. 1957, 79, 5318.

(2) Kelly, B. D.; Lambert, T. H. J. Am. Chem. Soc. 2009, 131, 13930.

(3) Vanos, C. M.; Lambert, T. H. Angew. Chem. Int. Ed. 2011, 50,12222

(4) Hardee, D. J.; Kpvalchuke, L.; Lambert, T. H. J. Am. Chem. Soc. 2010, 132, 5002 .
(5) Srivastava, V. P.; Patel, R. GarimaYadav, L. D. S. Chem. Commun. 2010, 46, 5808.

(6) Vanos, C. M.; Lambert, T. H. Chem. Sci. 2010, 1, 705.

(7) Kelly, B. D.; Lambert, T. H. Org. Lett. 2011, 13, 740.

(8) Perkins, W. C.; Wadsworth, D. H. Synthesis 1972, 205.

(9) Nogueira, J. M.; Nguyen, S. H.; Bennett, C. S. Org. Lett. 2011, 13, 2814. 